\title{
Ground and Meteorological Effects on Sound Propagation in the Atmosphere - Predictions and Measurements
}

\author{
Y.W. Lam \\ School of Acoustics and Electronic Engineering, University of Salford, Salford M5 4WT, England
}

(Received 30 September 1999; accepted 4 April 2000)

\begin{abstract}
Sound propagation in the atmosphere is inevitably affected by the meteorological conditions. Most existing standard calculation schemes do not have the ability to account for meteorological effects over non-flat grounds. Numerical methods such as that based on the Parabolic Equation can deal with complex meteorological conditions but are limited to slowly varying grounds. A heuristic ray tracing model can be used to approximate the meteorological effects but the validity of using a linear sound speed profile to represent complex real life meteorological conditions is still debatable. In this paper, predictions of sound propagation under strong meteorological influences are investigated. Predictions from the standard calculation schemes of ISO and CONCAWE, the heuristic ray tracing model and the Parabolic Equation model are compared with reallife measurements over smooth, flat ground as well as over complex grounds. The situations include the presence of rough, non-flat ground with an intervening hill and considerable meteorological conditions with wind speeds up to $10 \mathrm{~m} / \mathrm{s}$. As expected, the more sophisticated numerical models produced better predictions but there are also indications that the meteorological conditions changes significantly over the propagation path over a complex ground and the usual practice of monitoring the meteorological conditions at two heights at a single locations may not be sufficient.
\end{abstract}

\section{INTRODUCTION}

How sound propagates outdoors is an important consideration in many environmental noise assessments. An accurate prediction of the propagation is needed to enable a correct assessment of the noise impact of new industrial developments to existing communities, or to decide whether permission should be given for the development of residential estates near industrial areas. Outdoor sound propagation is affected by a large number of interacting environmental factors. The factors that have significant effects on the propagation of noise may be listed as follows:

1. Atmospheric Air Absorption

2. Geometrical Spreading (Point/Line/Plane/Directivity)

3. Barrier Attenuation (Infinite/Finite/Thick/Absorptive)

4. Ground and Terrain Effect

5. Meteorological Effect (Wind and Temperature Gradients)

6. Miscellaneous Effects - In-Plant Screening, Building Reflections, Forests etc.

Strictly speaking, air absorption is also a meteorological effect. However in most environmental noise calculations air absorption is generally treated separately from the turbulence and refraction effects caused by wind and temperature gradients. In here we adopt this convention and meteorological effects in this paper refer only to those caused by atmospheric turbulence and wind and temperature gradients.

Although the above factors are listed individually in the above, they in fact interact and combine to produce the overall effect on the noise propagation. Some of the simpler effects, such as the air absorption and geometrical spreading can be calculated individually without significant loss of accuracy, and their calculation is well established in standard calculation procedures, such as References ${ }^{\mathbf{1}, 2}$. For the others, the interactions can be significant and should be considered as a whole. Unfortunately the interactions are generally rather complex and cannot be modelled conveniently by empirical means. As a result these environmental factors are generally calculated individually and their interactions largely ignored in most standard calculation methods. Theoretical or numerical methods, such as the Fast Field Method ${ }^{3}$, the Parabolic Equation Method ${ }^{4}$ and the Integral Equation Method $^{5}$, are available for more detailed calculations. These theoretical methods are usually developed to tackle specific features of the propagation effect and are generally limited by two requirements: 1) idealised conditions that are difficult to meet in real life; and/or 2) detailed descriptions of the environment that are difficult to obtain in practice. This is particular true in the modelling of meteorological effects where sufficiently detailed meteorological condition data are hard to obtain. The usefulness of the theoretical methods are currently limited to the study of specific problems, and for the general calculation of environmental noise, empirical methods are still preferred. This paper investigates the possible benefits of the theoretical models over standard calculation methods. A series of outdoor measurements in a variety of sites with different ground terrain and meteorological conditions was made to investigate the interactions between ground, barrier and meteorological effects. The results were compared to predictions made by different calculation methods covering the whole spectrum of complexity.

\section{CALCULATION METHODS}

\subsection{Empirical Methods}

Standard calculation methods, such as CONCAWE ${ }^{1}$ and ISO 9613 Part $2^{2}$ (and its VDI, Dutch and NORDFORSK 